\title{
Cirugía de revascularización miocárdica de la arteria descendente anterior con arteria mamaria interna izquierda sin circulación extracorpórea: Experiencia en el largo plazo
}

\author{
Juan Carlos Bahamondes S, A belardo Silva V, \\ Juan Salman A, G ustavo Meriño S, Jean P. D roguett G. \\ Beating heart anterior descending \\ coronary artery grafting with left \\ internal mammary artery: \\ A long term follow up experience
}

Background: In recent years the use of left internal mammary artery (LMA) as a graft to anterior descending artery (DA) with the off pump technique has been associated with similar good long term results as with the on pump technique. Aim: To report the results of LMA to DA bypass grafting without extracorporeal circulation (EC) for isolated DA lesions. Patients and methods: Descriptive study of 80 patients subjected to coronary surgery between 1999 and 2007. Results: Mean age of patients was $63 \pm 10$ years and 60 were male. There was no operative mortality or stroke. One patient with a myocardial infarction required a reoperation. Actuarial survival was $98 \%$ at 97 months. Conclusions: In this group of patients the use of LMA as a coronary bypass graft to DA with the off pump technique is a safe surgical procedure, providing a prolonged cardiac event free survival (mortality, angina, myocardial infarction, and need for a new coronary procedure) Rev Méd Chile 2009; 137: 18-24).

(Key words: Coronary artery bypass, off-pump; Internal mammary-coronary artery anastomosis; Myocardial infarction)

Recibido el 13 de marzo, 2008. Aceptado el 1 de octubre, 2008.

Cirugía Cardiovascular, Centro Cardiovascular, Hospital Regional Temuco. Departamento de Cirugía, Facultad de Medicina, Universidad de La Frontera. Temuco, Chile.

L a cirugía de revascularización miocárdica con circulación extracorpórea ha sido la técnica quirúrgica estándar en el tratamiento de lesiones

Correspondencia a: Dr. Juan Carlos Bahamondes S. Departamento de Cirugía, Universidad de La Frontera. Av. Manuel Montt 112, 4o piso, Temuco. Fono-Fax: 45-325760.

E mail: jcbahamo@ufro.cl únicas de la arteria descendente anterior mediante el uso de un injerto de arteria mamaria interna, la que ha demostrado una permeabilidad mantenida en el largo plazo cercana a $90 \%$ a 10 años. Esto se ha relacionado con excelentes resultados clínicos, una mayor supervivencia en el largo plazo y una menor incidencia de eventos cardiacos tardíos, según diferentes series nacionales e internacionales ${ }^{1-6}$. 
La cirugía de revascularización miocárdica sin circulación extracorpórea ha tenido un gran desarrollo en la década pasada, lo cual permitió definir que grupos de pacientes se beneficien con esta técnica. Sin embargo, en los últimos años, muchos grupos cardioquirúrgicos han demostrado buenos resultados con una baja morbilidad y mortalidad en el período postoperatorio ${ }^{7-11}$.

Dado que el tratamiento de las lesiones obstructivas de la arteria descendente anterior sigue siendo controvertido y la angioplastia con stent es una alternativa terapéutica vigente ${ }^{12,13}$, es necesario determinar los resultados obtenidos con la revascularización miocárdica de la arteria descendente anterior con un injerto de arteria mamaria interna sin circulación extracorpórea en el largo plazo, y de esta forma poder decidir con bases científicas, qué estrategia es más eficaz en este tipo de lesiones en nuestros pacientes.

\section{PACIENTES Y MÉTODO}

Estudio descriptivo de 80 pacientes coronarios con lesión obstructiva única de la arteria descendente anterior, ubicada tanto en el tercio proximal como en el tercio medio, en quienes la indicación quirúrgica estuvo determinada por ser lesiones no susceptibles de angioplastia según criterio del cardiólogo intervencional. Los pacientes fueron intervenidos en forma consecutiva en el período comprendido entre noviembre de 1999 y diciembre de 2007 por cirugía cardiovascular del Hospital Regional de Temuco. La decisión de que la cirugía se llevara a cabo sin circulación extracorpórea fue tomada por el cirujano tratante. Se excluyen a los pacientes con patologías cardiovasculares concomitantes. Previo a la cirugía, a todos los pacientes se les solicitó firmar la solicitud de consentimiento informado.

Los datos fueron obtenidos de fichas clínicas, base de datos de cirugía cardiovascular y de protocolos operatorios. El seguimiento a largo plazo se realizó mediante controles clínicos en policlínico de cirugía cardíaca o de las consultas de médicos tratantes, contactos telefónicos; y además, se determinó fecha y causa de muerte en el registro civil. Se determinó las características angiográficas preoperatorias, infarto miocárdico reciente (definido como aquel menor a 15 días de evolución) y procedimientos de angioplastia realizados previo a la cirugía.

Se analizó el tiempo operatorio, complicaciones postoperatorias ocurridas como infarto miocárdico, accidente cerebrovascular, infección de herida esternal y sangrado postoperatorio que requirió de exploración quirúrgica, requerimientos transfusionales, complicaciones respiratorias, tiempo en ventilación mecánica, presencia de fibrilación auricular, estadía en unidad de cuidados intensivos y estadía total. Para la determinación de la mortalidad de la serie, se consideró como aquella ocurrida dentro de los 30 días de postoperatorio. En el seguimiento se determinó fecha y causa de muerte alejada, reaparición de angina, infarto miocárdico y la necesidad de una nueva angioplastia o cirugía de revascularización miocárdica.

Técnica quirúrgica. Durante el mismo período 1.850 pacientes fueron sometidos a cirugía de revascularización miocárdica exclusiva, correspondiendo esta serie a $4 \%$ del total de operaciones. Los pacientes recibieron mitad de dosis de heparina $(2 \mathrm{mg} / \mathrm{kg})$, se realizó esternotomía media convencional y disección de la arteria mamaria intema izquierda. Se utilizó magnificación óptica de 2,5-3,0 x. Una vez abierto el pericardio el corazón es desplazado hacia la línea media monitorizando cambios en el perfil hemodinámico y electrocardiográfico en forma continua. Una vez elegida la zona de anastomosis, se utilizó un estabilizador cardiaco mantenido en posición mediante un sistema aspirativo a $\pm 250 \mathrm{mmHg}$. En todos los pacientes se usó oclusión coronaria proximal con Prolene 5/0. Un shunt intraluminal fue utilizado según preferencia del cirujano durante la confección de la anastomosis, la cual fue llevada a cabo con sutura irreabsorbible $7 / 0$. Una vez completado el procedimiento, el efecto hepańnico fue revertido con mitad de dosis de protamina.

Estadística. Se utilizó estadística descriptiva con medidas de tendencia central y dispersión. El método de Kaplan-Meier se utilizó para el cálculo de supervivencia actuarial, con el programa Stata 9.2,.

\section{RESULTAdos}

La edad promedio de la serie fue 64,5 años ( $\mathrm{DE} \pm$ 9.575) con un rango entre 35 y 78 años, la 
distribución de factores de riesgo para enfermedad coronaria se describen en la Tabla 1.

En cuanto a las variables preoperatorios estudiadas, la lesión obstructiva de la arteria descendente anterior era proximal en la mayoría de los casos y estaba localizada en el tercio medio en menor proporción (Tabla 2). La mayoría de los pacientes debutaron con la presencia de angina inestable, y en menor proporción presentaron un infarto miocárdico. Sin embargo, todos los pacientes fueron operados antes de quince días de ocurrido el infarto miocárdico. Quince pacientes tenían una angioplastia previa en la arteria descendente anterior proximal con signos claros de reestenosis y síntomas de angina (Tabla 3). La cirugía fue llevada a cabo sin inconvenientes en todos los pacientes, no siendo necesaria la conversión a circulación extracorpórea durante el acto operatorio. Para mantener la perfusión miocárdica distal al sitio de la anastomosis, se utilizó un shunt intracoronario en 30 pacientes. No hubo mortalidad quirúrgica, accidente cerebrovascular, ni sangrado en la serie. Un paciente presentó un infarto miocárdico de pared anterior en las primeras horas del postoperatorio confirmado por elec-

Tabla 1. Factores de riesgo de enfermedad coronaria en pacientes operados sin CEC

\begin{tabular}{|lcl|}
\hline & $\mathbf{n}=\mathbf{8 0}$ & $\%$ \\
\hline Hipertensión & 77 & 96,2 \\
Diabetes & 30 & 37,5 \\
Dislipidemia & 43 & 53,7 \\
Tabaquismo & 29 & 36,2 \\
Sexo masculino & 60 & 75 \\
\hline
\end{tabular}

\section{Tabla 3. Características preoperatorias de pacientes operados sin CEC}

\begin{tabular}{|llc|}
\hline & $\mathbf{n}$ & $\%$ \\
\hline Angor inestable & 70 & 87,5 \\
Infarto miocárdico & 29 & 36,2 \\
Angioplastia ADA & 15 & 18,7 \\
\hline
\end{tabular}

trocardiograma y enzimas cardiacas en UCI cardiovascular, debido a una trombosis aguda del puente, siendo reintervenido en forma inmediata efectuándose una reanastomosis del puente de arteria mamaria a la arteria descendente anterior mediante circulación extracorpórea. El tiempo quirúrgico fue de 173 minutos $(\mathrm{DE} \pm 45,8)$ en promedio.

Los requerimientos transfusionales fueron mínimos, no hubo fibrilación auricular y mortalidad en el postoperatorio en nuestra serie. El tiempo en ventilación mecánica fue de $2 \mathrm{~h}$ en promedio. La estadía en UCI y hospitalaria fue acortada, lo cual se describe en la Tabla 4.

Seguimiento. El seguimiento fue completo en todos los pacientes, el cual fue en promedio de $54,5$ meses ( $\mathrm{DE} \pm 7,35)$ efectuándose controles periódicos. No hubo reaparición de angina, presencia de un infarto miocárdico, necesidad de una nueva revascularización ni mortalidad en la serie hasta el cierre del estudio.

La probabilidad de supervivencia actuarial es de $98 \%$ a 97 meses. La probabilidad actuarial de estar libre de otro procedimiento de revascularización es de 98\% a 97 meses (Figura 1).

Tabla 2. C aracterísticas angiográficas de pacientes operados sin CEC

\begin{tabular}{|lll|}
\hline & $\mathbf{n}$ & $\%$ \\
\hline Lesión ADA proximal & 70 & 87,5 \\
Lesión ADA media & 10 & 12,5 \\
\hline
\end{tabular}

ADA: Arteria descendente anterior

Tabla 4. M orbilidad y estadía hospitalaria en pacientes operados sin CEC

\begin{tabular}{|lc|}
\hline & LIM A D A sin CEC \\
\hline Transfusión (no GR) & $1 \pm 15,78(1-3)$ \\
Tiempo ventilación mecánica (h) & $2 \pm 21,8(1-6)$ \\
Fibrilación auricular & 0 \\
Mortalidad & 0 \\
Días UCI (días) & $1,7 \pm 35,7(1-3)$ \\
Días Total (días) & $5 \pm 56,21(4-9)$ \\
\hline
\end{tabular}




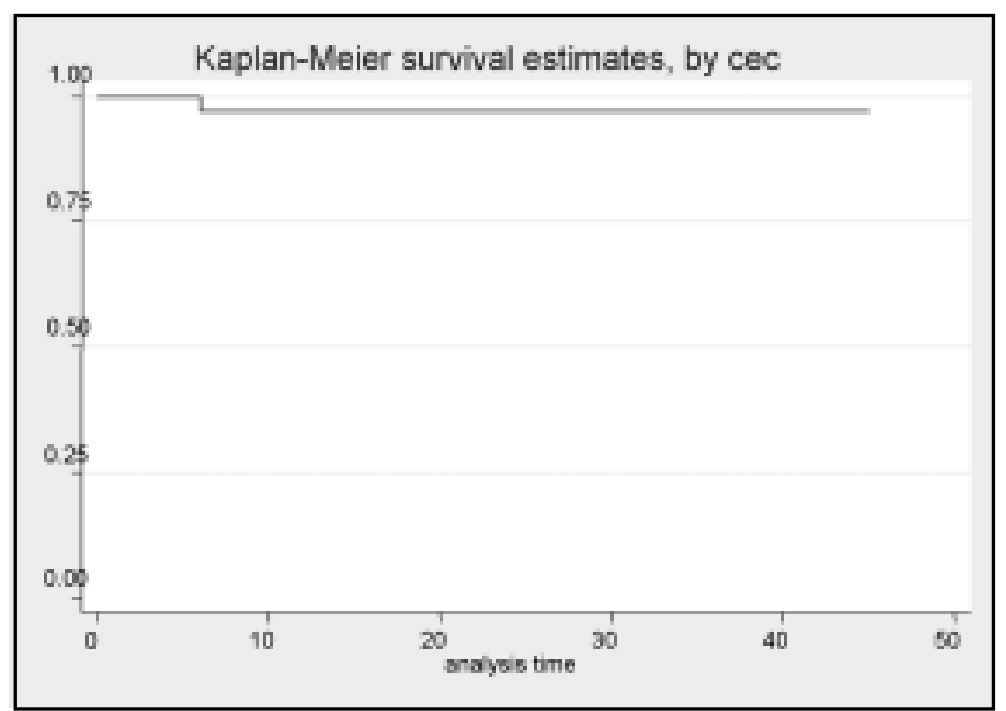

Figura 1. Probabilidad de sobrevida actuarial en pacientes con revascularización miocárdica sin CEC.

\section{Discusión}

La revasculanización de la arteria descendente anterior se ha efectuado en forma clásica mediante el uso de la arteria mamaria interna izquierda con circulación extracorpórea con excelentes resultados a nivel mundial ${ }^{1,6,14}$. En un estudio comparativo reciente efectuado por nuestro grupo en este tipo de pacientes no hubo diferencias estadísticamente significativas para angina, infarto miocárdico o necesidad de una nueva revascularización entre los grupos, con una probabilidad de sobrevida excelente para ambos a mediano plazo. Como tampoco hubo diferencias en el número de transfusiones efectuadas, presencia de fibrilación auricular, complicaciones neurológicas, tiempo en unidad de cuidados intensivos y estadía total ${ }^{15}$. Todo lo cual puede deberse a que se trata de una población de pacientes altamente seleccionada y de bajo riesgo comparativo en quienes la aorta no es manipulada. Sin embargo, se obtuvieron diferencias en el número de horas en ventilación mecánica a favor del grupo intervenido sin circulación extracorpórea. En relación a la relación costo-efectividad, el estudio de Ascione et $\mathrm{al}^{8}$ demostró diferencias estadísticamente significativas a favor de la cirugía sin circulación extracorpórea con menores costos operativos por paciente.

Por otra parte, el advenimiento del tratamiento por vía percutánea de las lesiones coronarias y el uso de stents intracomnarios permitió la incorporación de una altemativa terapéutica en la revascularización miocárdica ${ }^{16}$, pero con una incidencia significativa de síntomas recurrentes de hasta $30 \%$ de los pacientes debido a reestenosis evaluada mediante angiografía a pocos meses de seguimiento ${ }^{6,13}$. Todo lo cual significa la necesidad de un nuevo procedimiento de revascularización en el corto plazo, lo que en términos económicos significa un costo elevado de tratamiento, manteniéndose la reestenosis intrastent como un problema formidable y de alto impacto económico ${ }^{12,13}$. Además, nuevos estudios referentes al uso de stents con drogas citoestáticas demuestran resultados iniciales buenos pero con complicaciones en el seguimiento debido a la necesidad de utilizar terapia anticoagulante, agregando una mayor morbilidad asociada al procedimiento $12,13,16$.

La arteria descendente anterior proximal ha demostrado ser el sitio coronario de mayor reestenosis postangioplastia, presentándose hasta en $40 \%$ a 50\% de los pacientes ${ }^{9}$. Varios estudios han comparado los resultados de la angioplastia versus la cirugía coronania con circulación extracorpórea en estenosis proximal de la arteria descendente anterior, obteniendo una baja incidencia de infarto perioperatorio en el grupo quirúrgico, pero según diferentes series, con un porcentaje variado de oclusión aguda del vaso con infarto miocárdico y necesidad de cirugía de revascu- 
larización urgente en el grupo tratado mediante angioplastia. No se observa diferencias significativas en cuanto a mortalidad, pero la necesidad de una nueva revascularización es sustancialmente más alta en el grupo con angioplastia. Otros estudios demuestran retrospectivamente mejores resultados y calidad de vida en pacientes en quienes se utilizó la arteria mamaria interna, demostrando que los pacientes tienen mayor supervivencia libre de angina, infarto miocárdico y necesidad de nueva revascularización ${ }^{11-}$ ${ }^{16}$ como es posible observar en el estudio efectuado en nuestro centro y por el grupo de la Universidad Católica de Chile ${ }^{5,18-20}$, que a su vez son comparables con otros estudios a nivel mundial ${ }^{20-26}$.

Por otra parte, en los últimos años los avances en la técnica quirúrgica de la cirugía de revascularización miocárdica sin circulación extracorpórea en base a estabilizadores cardiacos han permitido reducir los riesgos en los grupos de pacientes cuyo estado preoperatorio es crítico ${ }^{27-29}$. Hoy en día, esta técnica constituye una alternativa quirúrgica conocida, alcanzando entre $20 \%$ a 30\% del total de cirugías coronarias a nivel mundial ${ }^{21,30,31}$. Algunos metaanálisis de estudios clínicos prospectivos y randomizados que comparan angioplastia versus cirugía coronaria sin circulación extracorpórea son claros en demostrar los beneficios superiores obtenidos a nivel mundial con esta técnica en términos de morbilidad y mortalidad y de un estado libre de eventos cardiovasculares en el largo plazo ${ }^{32-39}$. De lo cual se desprende que, aunque ambas técnicas mejoran el estado clínico, con un riesgo de muerte y

\section{REFERENCIAS}

1. Kolessov VI. Mammary artery-coronary artery anastomosis as a method of treatment for angina pectoris. J Thorac Cardiovasc Surg 1967; 54: 535-44.

2. Loop FD, Lytle BW, Cosgrove DM, Stewart RW, GooRmastic M, WiшAMs GW. Influence of the internal mammary graft of 10 year survival and other cardiac events. N Engl J Med 1986; 314: 1-6.

3. Buffolo E, De Andrade CS, Branco JN. Comonary artery bypass grafting without cardiopulmonary bypass. Ann Thorac Surg 1996; 61: 63-6.

4. Calafiore aM, Gianmarco G, Teodori G, Gaunna $\mathrm{S}$, Maddestra N, Paloscia L. Midterm results after mini- de infarto miocárdico similar en pacientes con enfermedad proximal de la arteria descendente anterior, la angioplastia está limitada en sus resultados por un número mayor de reintervenciones, lo cual no la hace una altemativa durable en el largo plazo. En nuestro medio, si bien es cierto existen publicaciones de diferentes grupos que demuestran ventajas evidentes de la cirugía frente a la angioplastia en el largo plazo 5,6,17,18, no existen comunicaciones de los resultados obtenidos con la estrategia sin circulación extracorpórea en este tipo de lesiones.

Como se ha demostrado en este estudio, la técnica empleada de injerto de arteria mamaria interna a la arteria descendente anterior sin circulación extracorpórea es una técnica extremadamente segura, sin mortalidad y prácticamente sin morbilidad asociada. Por este motivo, en base a la experiencia alcanzada y a los resultados comparativos obtenidos, la técnica empleada es el método de elección en el tratamiento de lesiones únicas de la arteria descendente anterior, salvo escasas excepciones como la presencia de una arteria descendente anterior intramiocárdica, todo lo cual se sustenta además en la combinación de una biología única entre el gran flujo sanguíneo diagonal y septal de la arteria descendente anterior y la arteria mamaria interna; la cual constituye un puente arterial de larga duración, que permite tiempos prolongados de supervivencia libre de eventos como angina, infarto miocárdico, mortalidad y de la necesidad de un nuevo procedimiento de revascularización en el largo plazo.

mally invasive coronary surgery (LAST operation). J Thorac Cardiovasc Surg 1998; 115: 763-77.

5. Garayar B, Irarrázaval MJ, Morán S, Zalaquett R, Becker P, Maturana G et al. Revascularización miocárdica de la arteria descendente anterior con anastomosis mamaria con técnica clásica. Rev Esp Cardiol 2000; 53: 316-20.

6. Bahamondes JC, Meriño G, Silva A, Salman J. Revascularizacion miocárdica de la arteria descendente anterior con arteria mamaria interna izquierda con circulación extracorpórea. Seguimiento a 10 años. Rev Méd Chile 2005; 133: 881-6.

7. Vural KM, Tasdemi O, Karagaz H, Emir M, Tarcon O, BAYAZIT K. Comparison of the early results of corona- 
ry artery bypass grafting with, and without extracorporeal circulation. J Thorac Cardiovasc Surg 1995; 43: 320-5.

8. Ascione R, Loyd CT, Underwood MJ. Economic Outcome of Off-Pump Coronary Artery Bypass Surgery: A Prospective Randomized Study. Ann Thorac Surg 1999; 68: 2237-42.

9. Bull DA, Neumayer LA, Stringham JC. Coronary artery bypass grafting with cardiopulmonary bypass versus off-pump cardiopulmonary bypass grafting: does eliminating the pump reduce morbidity and cost? Ann Thorac Surg 2001; 71: 170-5.

10. Berson AJ, Smith JM, Woods S. Off-Pump Versus OnPump Coronary Artery Bypass Surgery: Does the Pump Influence Outcome? J Am Coll Surg 2004; 102-8.

11. Baumgartner F, Yokohama T, Gheissari A. Effect of offpump Coronary Artery Bypass Grafting on Morbidity. Am J Cardiol 2000; 1021-2.

12. Goy JJ, Eeckhout E, Moret C, Burnand B, Vogt P, StAUFFER JC ET AL. Five year outcome in patients with isolated proximal left anterior descending coronary artery stenosis trated with angioplasty or left internal mammary artery grafting. A prospective trial. Circulation 1999; 99: 3255-9.

13. Versaci F, Gaspordone A, Tomai F, Crea F, Chiariello L, GiofFre P. A comparison of coronary artery stenting with angioplasty for isolated stenosis of the proximal left anterior descending coronary artery. N Engl J Med 1997; 336: 817-22.

14. Sergeant P, Biackstone E, Meyns B. Validation and interdependence with patients variables of the influence of procedural variables on early and late survival after CABG. Eur J Cardio Thorac Surg 1997; 12: 1-19.

15. Bahamondes JC. Silva A, Merino G et al. Revascularización miocárdica de la arteria descendente anterior con arteria mamaria interna izquierda: comparación de dos técnicas en el largo plazo. Rev Chil Cir 2007; 59: 330-6.

16. Fischman DL, Leon MB, Baim DS, Schatz R, Savage $\mathrm{M}$, PENN. A randomized comparison of coronary stent placement and balloon angioplasty in the treatment of coronary disease. N Engl J Med 1994; 331: 496-501.

17. Morán S, Irarrázaval MJ, Zalaquett R, Vilavicencio M, Garayar B, MuÑoz C ET aL. Revascularización miocárdica con arteria mamaria interna bilateral. Rev Chil Cardiol 1996; 15: 4-8.

18. Morán S, Irarrázaval MJ, Zalaquett R, Vilavicencio M, Garayar B, MuÑoz C et al. Revascularización miocárdica con una y dos arterias mamarias: resultados clínicos y seguimiento alejado. Rev Méd Chile 1997; 125: 391-401.
19. Morán S, Larraín E, Becker P, Irarrázaval MJ, Urzúa J, LEMA G ET AL. Evolución de las indicaciones, técnica quirúrgica y resultados de la revascularización miocárdica. Rev Chil Cardiol 1993; 12: 13-7.

20. Michaux I, FiLPovic M. Effects of on-pump versus offpump coronary bypass graft surgery on right ventricular function. J Thorac Cardiovasc Surg 2006; 131: 1281-8.

21. Cuenca J, Bonome C. Cirugía coronaria sin circulación extracorpórea y otras técnicas mínimamente invasivas. Rev Esp Cardiol 2005; 58: 1335-48.

22. Geert JM, VAn Der HeiJden. Meta-analysis on the effect of off-pump coronary bypass surgery. Eur J Cardiothorac Surg 2004; 81-4.

23. Puskas J, Wiwams W, Duke P, Stapie JR, Glas Ke, MARSHAL JJ. Off-pump coronary artery bypass grafting provides complete revascularization with reduced myocardial injury, transfusion requirements and length of stay: a prospective randomized comparison of two hundred unselected patients undergoing off-pump versus conventional coronary artery bypass grafting. J Thorac Cardiovasc Surg 2003; 125: 797-808.

24. RASTAN AJ, BITTNER H, GUMmert JF. On-pump beating heart versus off-pump coronary artery bypass surgery-evidence of pump-induced myocardial injury. Eur J Cardiothorac Surg 2005; 27: 1057-64.

25. Tabata M, Takanashi SH, Fukui T. Off-Pump Coronary Artery Bypass Grafting in Patients with Renal Disfunction. Ann Thorac Surg 2004; 78: 2044-9.

26. Lee J, Lee SJ, Tsushima WT. Benefits of Off-Pump bypass on Neurologic and Clinical Morbidity: A prospective Randomized Trial. Ann Thorac Surg 2003; 76: 18-26.

27. Racz MJ, Hannan E, Isom W. A Comparison of Short and Long-Term Outcomes After Off-Pump and OnPump Coronary Artery Bypass Graft Surgery With Sternotomy. J Am Coll Cardiol 2004; 43: 557-64.

28. Locker C, Shapira I, Paz Y, Kramer A, Gurevitch J, MATSA M. Emergency myocardial revascularization for acute myocardial infarction: survival benefits of avoiding cardiopulmonary bypass. Eur J Cardiothorac Surg 2000; 17: 234-8.

29. SterniK L, MoshKovitz Y, Hod H, Mohr R. Comparison of myocardial revascularization without cardiopulmonary bypass to standard open heart technique in patients with left ventricular dysfunction. Eur J Cardiothorac Surg 1997; 11: 123.

30. Herrera JM, Cuenca J, Campos V, Rodríguez F, Vaie JV, Jufré A. Cinugía coronaria sin circulación extracomórea: 5 años de experiencia. Rev Esp Cardiol 1998; 51: 136-40.

31. Omeroglu S, Kirali K, Guler M, Toker M, Ipek G, Isik $\mathrm{O}$. Midterm angiographic assessment of coronary artery bypass grafting without cardiopulmonary bypass. Ann Thorac Surg 2000; 70: 844-50. 
32. Cheng DC, Bainbridge D, Martin J. Does Off-Pump Coronary Artery Bypass Reduce Mortality, Morbidity, and Resource Utilization When Compared with Conventional Coronary Artery Bypass? A Meta-analysis of Randomized Trials. Anesthesiology 2005; 102: 188-203.

33. Al-Ruzzeh S, George S, Bustami M, Wray J, Ilsley C, Athanasiou T, AmRan M. Effect of off-pump coronary artery bypass surgery on clinical, angiographic, neurocognitive, and quality of life outcomes: randomised controlled trial. BMJ 2006; 332: 1365-70.

34. Van Dijk D, Nierich A, Jansen E. Early Outcome After Off-Pump Versus On-Pump Coronary Bypass Surgery. Circulation 2001; 104: 1761-6.

35. Bair TL, Muhlestein JB, May HT, Meredith KG, Horne BD, Pearson RR et aL. Surgical revascularization is associated with improved long-term outcomes compared with percutaneous stenting in most subgroups of patients with multivessel coronary artery disease: results from the Intermountain Heart Registry. Circulation 2007; 116 (11 Suppl): I226-31.

36. JafFery Z, Kowalski M, Weaver WD, Khanal S. A metaanalysis of randomized control trials comparing minimally invasive direct coronary bypass grafting versus percutaneous coronary intervention for ste- nosis of the proximal left anterior descending artery. Eur J Cardiothorac Surg 2007; 31: 697.

37. Drenth DJ, Winter JB, Veeger NJ, Monnink SH, Van Boven AJ, Grandjean JG et aL. Minimally invasive coronary artery bypass grafting versus percutaneous transluminal coronary angioplasty with stenting in isolated high-grade stenosis of the proximal left anterior descending coronary artery: six months' angiographic and clinical follow-up of a prospective randomized study. J Thorac Cardiovasc Surg 2002; 124: 103-5.

38. Takagi H, Tanabashi $\mathrm{T}$, Kawai N, Umemoto T. A metaanalysis of minimally invasive coronary artery bypass versus percutaneous coronary intervention with stenting for isolated left anterior descending artery disease is indispensable. J Thorac Cardiovasc Surg 2007; 134: 548.

39. Bainbridge D, Cheng D, Martin J, Novick R. Evidencebased Peni-operative Clinical Outcomes Research (EPiCOR) Group. Does off-pump or minimally invasive coronary artery bypass reduce mortality, morbidity, and resource utilization when compared with percutaneous coronary intervention? A meta-analysis of randomized trials. J Thorac Cardiovasc Surg 2007; 133: 623-31. 